\section{Depuis une quinzaine}

d'années, les physiciens

ont développé des techniques

de manipulations d'atomes

par laser qui permettent

de refroidir, de piéger

et d'observer un seul atome

à la fois. Le piégeage

d'atomes individuels se fait

à l'aide d'une pince optique,

terme qui désigne un laser

focalisé sur une tache

d'un micromètre de taille,

au foyer duquel se trouve

piégé l'atome.

Grâce à des méthodes

holographiques il est aussi

possible de produire

des matrices de pinces

optiques dont la géométrie

est pratiquement arbitraire.

On obtient ainsi des plans

d'atomes séparés de quelques

micromètres, arrangés selon

des géométries variées.

Au-delà de leur aspect

esthétique, ces méthodes

trouvent leurs applications

dans le domaine en pleine

expansion des technologies

quantiques.

\title{
Des pinces optiques pour piéger des atomes un par un...
}

Antoine Browaeys (antoine.browaeys@institutoptique.fr)

Laboratoire Charles Fabry, Institut d’0ptique, 2 avenue Augustin Fresnel, 91127 Palaiseau Cedex

En 1955, Erwin Schrödinger, l'un des fondateurs de la physique quantique, affirmait : "Nous ne faisons jamais d'expérience avec une seule particule... Dans des expériences de pensée, nous le faisons parfois, mais cela conduit invariablement à des conséquences ridicules » [1]. Moins de 20 ans plus tard, Hans Dehmelt démontrait le piégeage d'un seul électron en utilisant des champs électriques et magnétiques [2]. Dehmelt avait une idée simple en tête : en isolant un seul électron, on peut faire des mesures sur ses propriétés sans que celles-ci soient modifiées par d'autres électrons environnants. On accède ainsi à ses propriétés intrinsèques. Par ailleurs, puisque la particule est piégée, on peut faire la mesure pendant longtemps, ce qui augmente sa précision. Dehmelt parvint ainsi à mesurer le moment magnétique de l'électron avec une précision de onze chiffres significatifs, fournissant un test très poussé de l'électrodynamique quantique qui prévoit sa valeur.

Les physiciens se sont ensuite attachés à élargir le nombre et le type de particules que l'on peut piéger avec des champs électromagnétiques. En 1980, H. Dehmelt et $\mathrm{P}$. Toschek piégèrent ainsi un seul ion baryum. Mais le piégeage des atomes est beaucoup plus ardu que celui des ions. Les atomes sont neutres et, par conséquent, très peu sensibles aux champs électriques et magnétiques. Pour les ions, quelques volts sur une électrode suffisent à produire des pièges dont la profondeur est de quelques $10000 \mathrm{~K}$ $(\sim 1 \mathrm{eV})$ : cela signifie que des ions qui possèdent une énergie cinétique résiduelle correspondant à la température ambiante (300 K) peuvent y rester confinés. Pour les atomes, comme nous allons le voir, les pièges que l'on sait créer au laboratoire à partir de faisceaux laser ont des profondeurs de seulement quelques millikelvins! Pour y piéger un atome, il faut donc que celui-ci soit issu d'un gaz refroidi à une température au moins aussi basse ! Ce défi a été relevé au cours des années 1980 - 1990 grâce aux méthodes de refroidissement d'atomes par laser [3]. Ces techniques, que nous ne détaillerons pas ici, permettent de refroidir un gaz d'atomes (par exemple des atomes alcalins, tels que le sodium, le rubidium ou le césium) initialement proche de la température ambiante (entre 300 et $500 \mathrm{~K}$ ), jusqu'à des températures inférieures à 100 microkelvins ! Ces développements spectaculaires ont valu à W.D. Phillips, C. CohenTannoudji et S. Chu le prix Nobel de physique en 1997 [4].

\section{Principe de la pince optique}

Parmi le nombre considérable de travaux que le refroidissement d'atomes par laser a permis, il y a donc le piégeage optique d'atomes. Le principe peut en être compris par le modèle classique suivant. Le champ électrique $\mathrm{E}$ du laser polarise l'atome en séparant le barycentre des électrons de celui du noyau qui reste essentiellement immobile, car beaucoup plus lourd que les électrons. Cette séparation des charges induit un dipôle électrique $\mathbf{p}=\varepsilon_{0} \alpha \mathrm{E}\left(\varepsilon_{0}\right.$ est la permittivité du vide et $\alpha$ la polarisabilité de l'atome) qui interagit avec le champ du laser. L'énergie d'interaction résultante, moyennée sur une période d'oscillation du champ électrique, est $\mathrm{U}=-\langle\mathrm{p} . \mathrm{E}\rangle / 2=-\varepsilon_{0} \alpha\left\langle\mathrm{E}^{2}\right\rangle / 2$.

Cela signifie que cette énergie d'interaction est grande en valeur absolue là où le champ du laser est le plus intense, c'est-à-dire au voisinage d'un point de focalisation de celui-ci. La polarisabilité $\alpha$ de l'atome dépend de la différence entre la fréquence $\omega$ du laser et celle de la transition (ou résonance) atomique $\omega_{0}$ choisie pour l'expérience. Dans le cas où 
le laser est accordé à une fréquence inférieure à celle de la résonance atomique $\left(\omega<\omega_{0}\right)$, le champ électrique varie suffisamment lentement pour que le dipôle induit soit en phase avec lui, et de ce fait $\alpha>0$. Ainsi, un point de focalisation d'un laser constitue un minimum d'énergie pour l'atome et peut être un piège puisque cette énergie augmente (l'intensité diminuant) dès que l'on s'écarte du foyer.

Dans tout ce raisonnement nous avons ignoré l'émission spontanée par l'atome induite par le laser de piégeage. Cette approche est valable dès lors que la fréquence du laser est assez éloignée de celle de la résonance atomique. On montre ainsi que le taux d'émission spontanée varie comme $1 /\left|\omega-\omega_{0}\right|^{2}$, alors que la polarisabilité (et donc l'énergie d'interaction atome-laser) varie comme $1 /\left|\omega-\omega_{0}\right|$ : il est par conséquent possible de rendre négligeable l'émission spontanée par un atome, tout en conservant une valeur importante de l'énergie d'interaction entre l'atome et le laser. Dans ce régime, l'action du laser sur l'atome se résume donc à créer un potentiel conservatif (sans dissipation d'énergie).

Une pince optique est un piège optique basé sur le principe décrit ci-dessus, dans la situation particulière où le faisceau laser est focalisé sur une taille de l'ordre de la longueur d'onde $\lambda$ du laser. Dans ce cas-là, la longueur caractéristique de variation de l'intensité le long de l'axe de propagation (c'est la longueur de Rayleigh pour un faisceau gaussien) est aussi de l'ordre de grandeur de la longueur d'onde, si bien que le volume du piège est de l'ordre de $\lambda^{3}$. Pour un laser opérant autour de $1 \mu \mathrm{m}$, cela conduit à un volume de $1 \mu^{3}$. Par exemple, un atome de rubidium a une longueur d'onde de résonance autour de $780 \mathrm{~nm}$. Si on envoie un laser de longueur d'onde

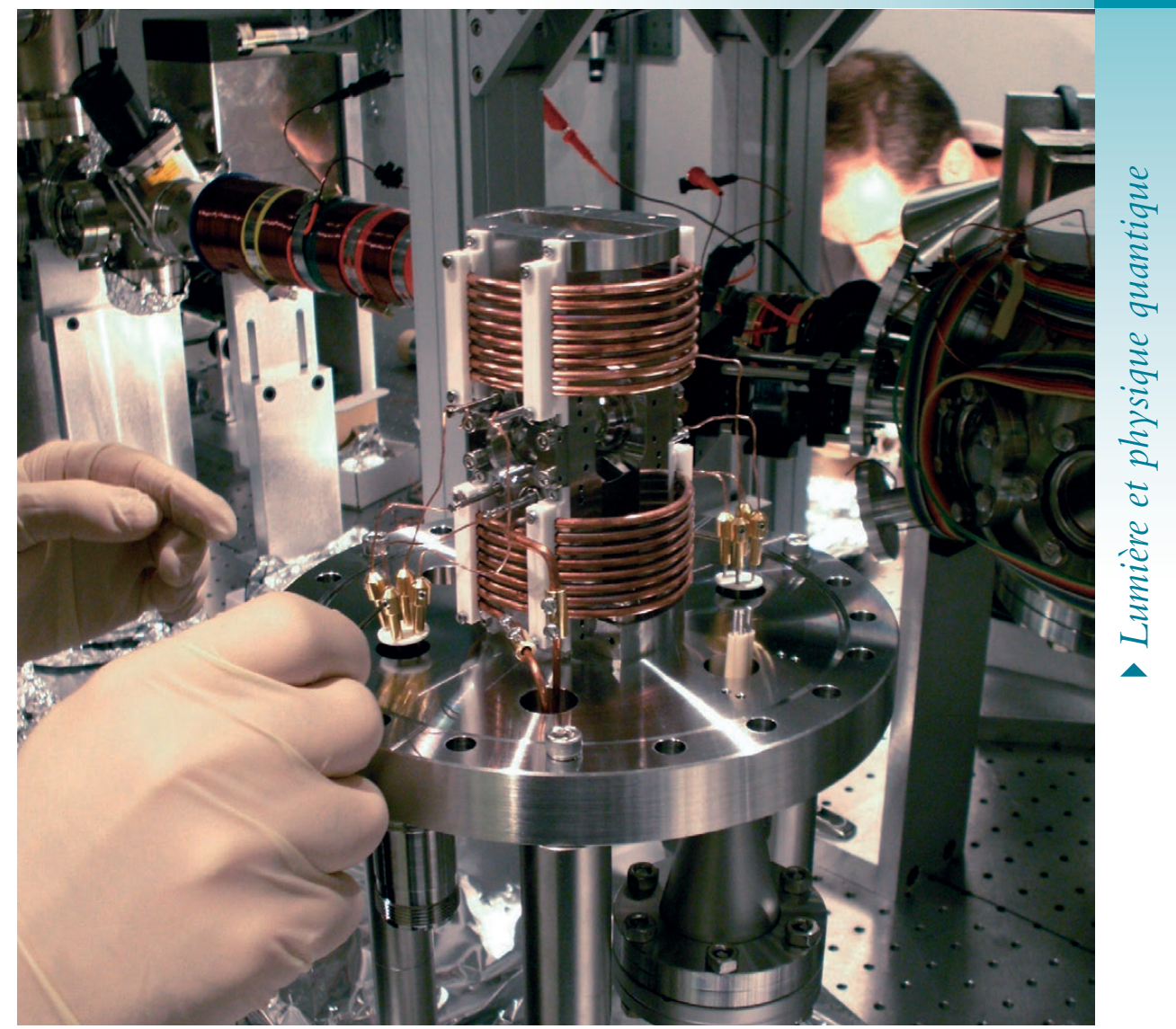

Partie centrale du dispositif expérimental de piégeage d'atomes individuels. La bride métallique supporte des bobines en cuivre permettant de produire le champ magnétique destiné à piéger et refroidir un nuage d’atomes de rubidium, ainsi que le support des lentilles asphériques (pièces métalliques verticales entre les deux bobines). La position du piège se trouve au centre de ce dispositif, entre les deux lentilles asphériques en regard (dont on aperçoit une partie). L'ensemble, dont la photo a été prise au cours du démontage final, sera placé dans l'enceinte à vide, dont on aperçoit une partie à droite de la photo.

$850 \mathrm{~nm}$ et de $5 \mathrm{~mW}$ de puissance (la puissance typique d'un pointeur laser...), focalisé sur $1 \mathrm{\mu m}^{2}$, la profondeur du potentiel ainsi créé - la pince optique vaut environ 1 millikelvin, comme annoncé plus haut.

\section{La pince optique en pratique}

Focaliser de la lumière sur une tache (un «spot») de l'ordre de grandeur de la longueur d'onde de la lumière n'est pas si facile. En effet, la taille minimale $w$ du spot est fixée par la limite de diffraction de la lentille utilisée : si celle-ci possède une ouverture numérique $\mathrm{ON}^{(\mathrm{a})}$, $w=1,22 \lambda /(2 \mathrm{ON})$. Pour une lentille ouverte à $\mathrm{ON}=0,5, w \approx 1 \mu \mathrm{m}$ pour $\lambda=850 \mathrm{~nm}$. Pour atteindre la limite de diffraction, la lentille ne doit pas présenter d'aberrations. C'est techniquement d'autant plus difficile à obtenir que son ouverture numérique est grande. Bien sûr, on trouve dans le commerce des objectifs de microscope d'excellente qualité avec des ouvertures numériques proches de 1, mais en général au prix d'une distance de travail petite et, de plus, comportant de nombreuses lentilles accolées que l'on ne peut pas placer facilement sous vide, comme il est souvent nécessaire de le faire pour manipuler des atomes froids. Même si la première réalisation du piégeage d'atomes individuels (voir ci-dessous) a été obtenue avec un objectif à plusieurs lentilles spécialement conçu à cet effet, ce piégeage s'est vraiment développé grâce aux progrès spectaculaires de la technologie des lentilles dites asphériques, c'est-à-dire ayant la bonne forme pour opérer à la limite de diffraction. Ce développement a été motivé par la lecture optique des compact discs car plus la taille du faisceau de lecture est petite, plus on peut coder d'information sur le disque. De nos jours, on trouve dans le commerce, pour quelques dizaines d'euros, des lentilles de $1 \mathrm{~cm}$ de focale (autorisant ainsi une distance de travail plus convenable) et d'ouverture numérique 0,5 , exactement ce qu'il nous faut pour produire une pince optique! 

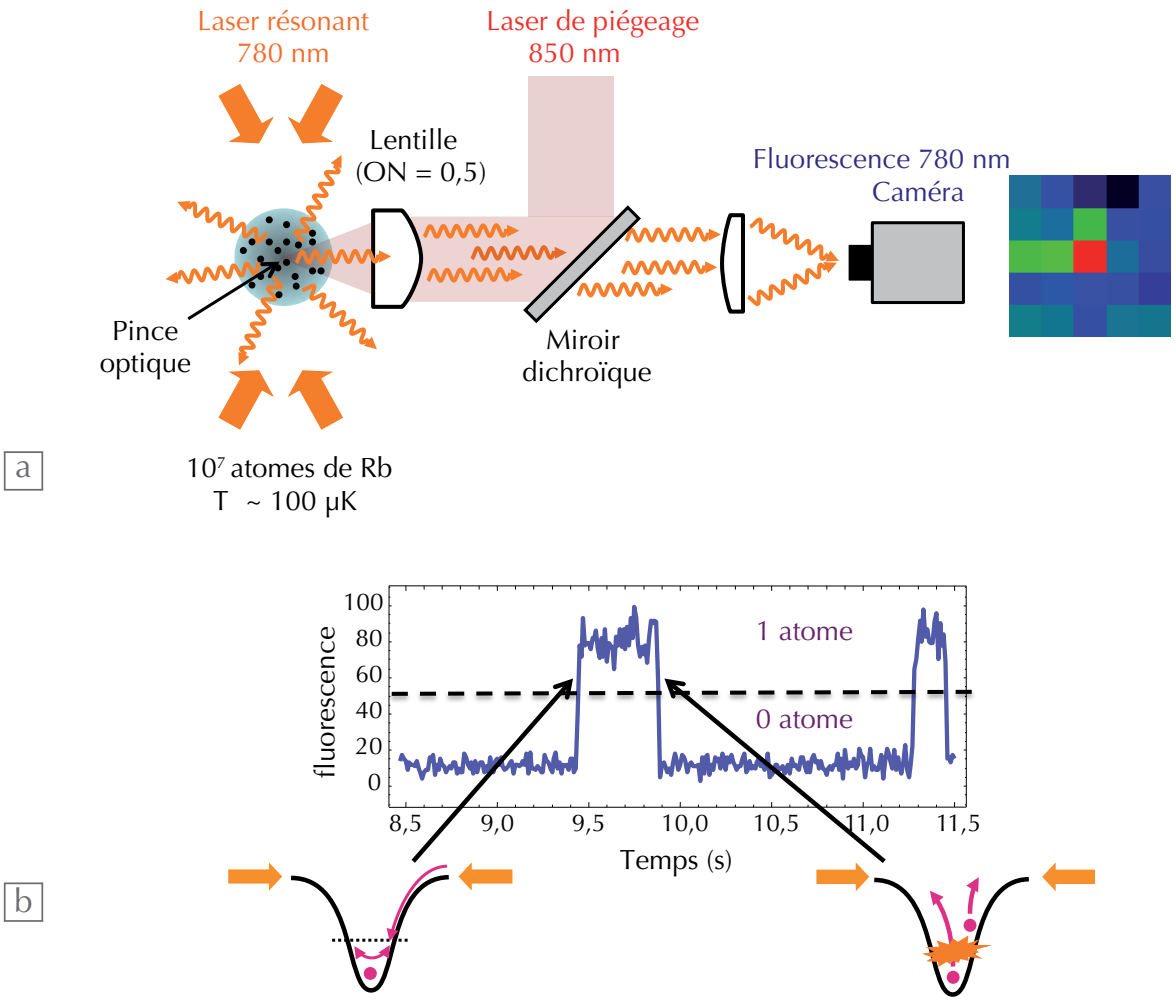

1. Principe d'une pince optique pour le piégeage d'atomes.

(a) Schéma du montage optique. La pince est produite par un faisceau laser à $850 \mathrm{~nm}$, focalisé par une lentille asphérique d'ouverture numérique 0,5. Un nuage d'atomes de rubidium refroidis par laser, placé au voisinage du foyer de la lentille, sert de réservoir pour charger la pince. La fluorescence émise par les atomes situés dans la région du piège est collectée sur un pixel d’une caméra. La fluorescence apparaît en rouge sur le pixel.

(b) Signal obtenu sur le pixel (en rouge sur la figure a) collectant la lumière provenant de la zone de piégeage en fonction du temps. La brusque augmentation du signal indique l'entrée d'un atome dans la pince (schématisée par le puits de potentiel en bas à gauche), ralenti par les lasers de refroidissement. La brusque disparition correspond à l'entrée d'un deuxième atome dans la pince, suivie d'une collision très rapide catalysée par les lasers à $780 \mathrm{~nm}$, qui expulse les deux atomes et vide donc le piège (schéma en bas à droite).

\section{Piégeage d'atomes individuels}

Nous avons maintenant tous les ingrédients pour comprendre le piégeage d'atomes dans une pince optique et la manière dont nous les observons. Nous réalisons pour cela un montage optique qui porte le nom de " microscopie confocale ", très utilisé par exemple en biologie. Il permet de focaliser un faisceau sur une petite tache et de collecter la lumière émise par les atomes situés au foyer. Comme la même lentille est utilisée pour collecter cette lumière, la résolution spatiale est donc égale à la taille de la tache de la pince optique (fig. 1a). Une lentille asphérique est éclairée par un faisceau laser à une longueur d'onde de $850 \mathrm{~nm}$, créant ainsi une pince optique de taille micrométrique. Autour de ce point focal, on produit un nuage d'atomes froids par refroidissement laser. Typiquement, nous collectons dix millions d'atomes de rubidium dans un volume de $1 \mathrm{~mm}^{3}$ autour de la pince optique. Ce refroidissement laser implique la présence de faisceaux laser à une longueur d'onde très proche de celle de la résonance atomique du rubidium $(\lambda=780 \mathrm{~nm})$. Un des atomes de ce nuage d'atomes froids entre alors à un instant aléatoire dans la pince optique, tout en étant freiné par les faisceaux de refroidissement, et y reste piégé.

Pour observer la présence de l'atome dans la pince, nous collectons avec la même lentille la lumière de fluorescence émise par cet atome soumis en permanence au laser à $780 \mathrm{~nm}$, qui a donc une double fonction : il sert à la fois à ralentir les atomes, mais aussi à éclairer l'atome qui diffuse la lumière. À l'aide d'un miroir dichrö̈que séparant la lumière à $850 \mathrm{~nm}$ de celle de fluorescence à $780 \mathrm{~nm}$, nous envoyons celle-ci sur une caméra en faisant en sorte que toute la lumière provenant de la zone du piège se retrouve sur un pixel de la caméra.
De cette manière on peut "voir " un atome, dans son acception la plus littérale, l'œil de l'observateur étant remplacé par une caméra par simple commodité ! La variation de la quantité de lumière reçue sur le pixel de la caméra en fonction du temps est montrée sur la figure $1 \mathrm{~b}$. Nous constatons que partant d'un niveau de lumière très faible correspondant au bruit de fond de l'expérience, le signal augmente soudainement. Nous attribuons ce saut au piégeage d'un atome dans la pince. L'absence de double palier traduit le fait que nous ne piégeons pas deux atomes à la fois. En effet, lorsqu'un deuxième atome entre dans la pince, la lumière à $780 \mathrm{~nm}$ catalyse une collision très violente entre les deux atomes, qui les expulse pratiquement instantanément en dehors du piège. Le fait que cette collision soit très rapide provient du volume très faible de la pince optique. Cette source d'atomes individuels est toutefois non déterministe : nous ne pouvons pas 
prévoir quand l'atome entre dans la pince. Cependant, dès qu'il est piégé, il signale sa présence par sa fluorescence. Nous pouvons alors placer un seuil sur la quantité de lumière détectée et attribuer un signal haut à la présence d'un atome. Si par la suite nous éteignons tous les faisceaux laser à $780 \mathrm{~nm}$, ne conservant que le faisceau de la pince optique, nous pouvons conserver l'atome pendant près d'une dizaine de secondes avant qu'il ne s'échappe naturellement du piège ${ }^{(b)}$ ! C'est amplement suffisant pour réaliser des expériences avec cet atome, car en général celles-ci prennent moins de $100 \mathrm{~ms}$ !

La démonstration du piégeage d'atomes uniques par la méthode décrite ci-dessus a été obtenue pour la première fois en 2000 à l'Institut d'Optique par Philippe Grangier et son équipe, au cours de la thèse de doctorat de Nicolas Schlosser, en utilisant un objectif spécialement conçu par les ateliers d'optique de l'Institut [5]. Depuis, en 2007, nous avons démontré, toujours à l'Institut d'Optique, cette méthode en utilisant une simple lentille asphérique commerciale [6]. Une dizaine de groupes de par le monde a maintenant reproduit notre configuration expérimentale.

\section{Fabrication de matrices de pinces : vers des cristaux artificiels d'atomes à deux dimensions}

Pour beaucoup d'applications (voir cidessous), un seul atome ne suffit pas. Par exemple, de nombreux groupes essaient de contrôler les interactions entre quelques atomes arrangés dans des géométries bien définies. Le montage expérimental décrit plus haut se prête très bien à l'obtention de matrices de pinces optiques de configuration arbitraire.

Pour produire ces matrices, on utilise la diffraction : sur le trajet du faisceau laser de la pince optique on insère un modulateur de phase spatiale. Il s'agit d'un dispositif à base de cristaux liquides dont l'indice optique $n(\mathrm{x}, \mathrm{y})$ peut varier d'un point $(\mathrm{x}, \mathrm{y})$ à un autre du plan perpendiculaire à la direction de propagation de la lumière, en fonction d'une tension localement appliquée. Un faisceau lumineux de longueur d'onde $\lambda$ qui traverse ce dispositif d'épaisseur $L$ voit sa phase spatiale modulée d'une quantité

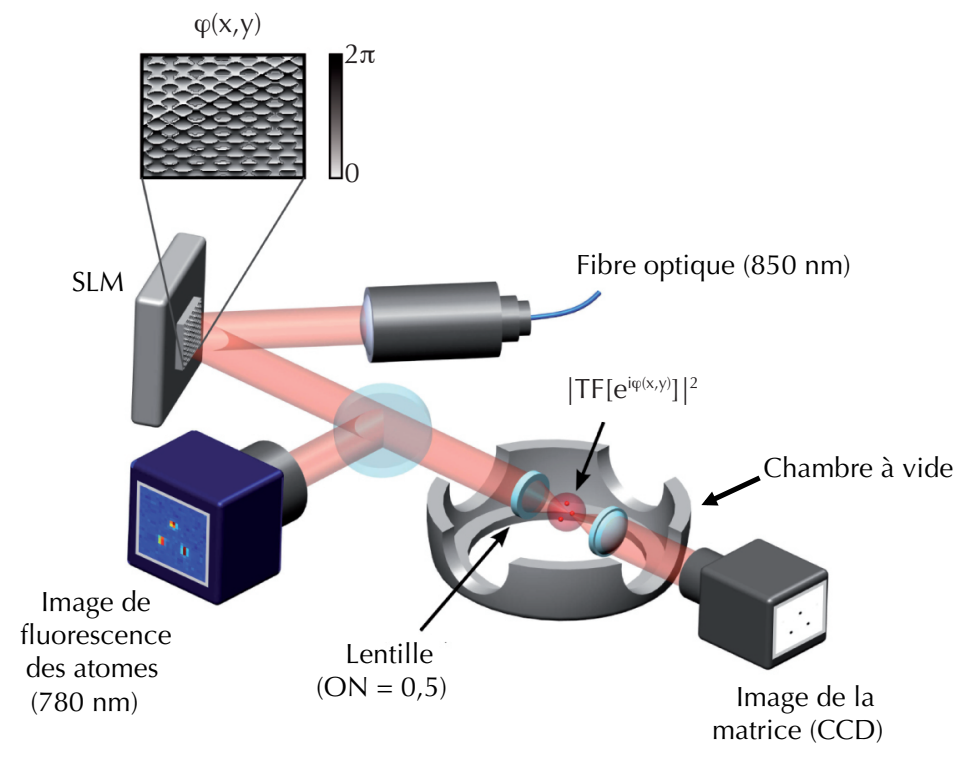

a

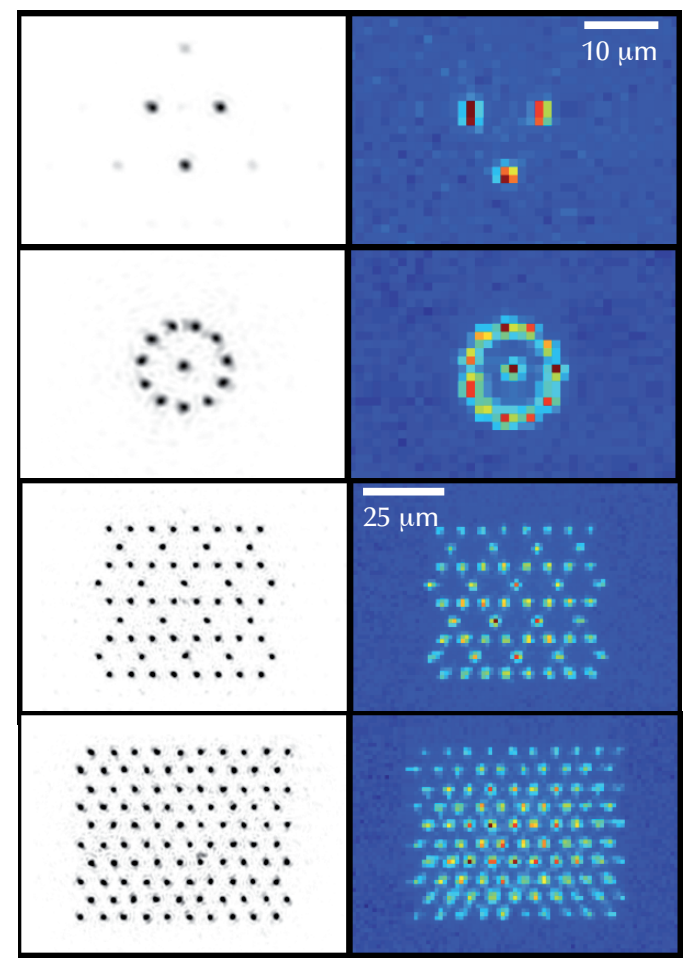

2. Production de matrices de pièges optiques.

(a) Dispositif expérimental. Un modulateur de phase spatiale (SLM) imprime une phase $\varphi(x, y)$ sur le faisceau laser $(\lambda=850 \mathrm{~nm})$ servant au piégeage. Celui-ci est focalisé par une lentille très ouverte. À son foyer, on obtient la figure de diffraction du facteur de phase. En ajustant cette phase, on produit des matrices de pièges de taille micrométrique avec différentes géométries. Chaque piège contient un seul atome dont on détecte la présence par la lumière de fluorescence induite par un laser résonant avec une transition atomique (ici $\lambda=780 \mathrm{~nm}$ ).

(b) Colonne de gauche : répartitions d'intensité dans le plan focal, mesurées par le capteur CCD situé après la chambre à vide. Colonne de droite : images de fluorescence pour des matrices de pièges contenant au plus un seul atome. Chacune des images est la somme de nombreuses réalisations de la même configuration de piégeage. 
$\varphi(\mathrm{x}, \mathrm{y})=2 \pi[n(\mathrm{x}, \mathrm{y})-1] L / \lambda$. La théorie de la diffraction enseigne que dans le plan focal de la lentille, la répartition d'intensité est le module carré de la transformée de Fourier du facteur de phase $e^{i \varphi(x, y)}$. Ainsi, pour obtenir une répartition d'intensité de géométrie donnée au foyer de la lentille, il suffit de programmer le modulateur de phase pour qu'il génère la modulation de phase $\varphi(x, y)$ adéquate.

Le problème est en fait un peu plus compliqué qu'il n'y parait : la théorie de la diffraction indique que ce sont les répartitions de champ électrique avant la lentille et dans son plan focal (et non les intensités associées) qui sont transformées de Fourier l'une de l'autre. La donnée de l'intensité du champ dans le plan focal de la lentille ne suffit donc pas : il manque l'information sur la phase pour pouvoir calculer la répartition de champ électrique au niveau du modulateur de phase. D'autre part, la solution d'un tel problème conduit à une répartition de champ avant la lentille modulée à la fois en phase et en amplitude, ce qu'un modulateur de phase pur ne peut évidemment pas réaliser... Le problème a toutefois été résolu dès les années 1970 par le développement d'algorithmes permettant de calculer une phase $\varphi(x, y)$ qui donne une répartition d'intensité approchée, mais arbitrairement proche de celle souhaitée dans le plan focal de la lentille.

Nous avons donc installé sur notre expérience un tel modulateur de phase pour produire des matrices de pinces optiques [7]. Le schéma optique est représenté sur la figure $2 \mathrm{a}$. La figure $2 \mathrm{~b}$ montre sur la colonne de gauche quelques exemples de répartition d'intensité obtenus dans le plan focal de la lentille. Lorsque cette matrice de pinces est focalisée dans le nuage d'atomes froids, nous observons sur la lumière de fluorescence que les pièges «clignotent " du fait de l'entrée aléatoire des atomes dans les pinces. Lorsque le nombre de pinces est faible (moins d'une dizaine en pratique), nous attendons que tous les pièges soient remplis pour démarrer une expérience. Si le nombre de pinces dépasse la dizaine, il devient peu probable d'avoir tous les pièges remplis avec un atome et la matrice présente donc des "trous " (en général de l'ordre de $30 \%$ du nombre de sites). On connait toutefois leur position exacte puisqu'ils apparaissent comme une absence de lumière de fluorescence à la position attendue du piège. Néanmoins, en prenant de nombreuses images successives et en les regroupant, on obtient les images montrées sur la colonne de droite de la figure $2 \mathrm{~b}$, sur laquelle tous les pièges ont l'air remplis. Notons que des méthodes ont été récemment suggérées pour augmenter la proportion de pièges remplis à un instant donné.

\section{Quelques applications des matrices d'atomes}

On dispose ainsi par cette méthode de plans d'atomes, arrangés suivant des géométries arbitraires et contrôlables. Les atomes sont séparés les uns des autres de plusieurs micromètres et il est donc relativement aisé de manipuler un atome particulier au sein de la matrice, par exemple à l'aide d'une pince optique supplémentaire que l'on peut focaliser sur un site spécifique de la matrice.

Les arrangements de la figure $2 \mathrm{~b}$ présentent une certaine similarité avec les matrices cristallines rencontrées en physique du solide. Cette remarque indique que ce système peut servir à simuler des situations de matière condensée. Par exemple, à l'aide de lasers on peut contrôler les interactions entre les atomes. Pour ce faire, notre groupe utilise deux états de l'atome de rubidium, l'un fondamental et l'autre très excité, appelé état de Rydberg ${ }^{(\mathrm{c})}$; les états de Rydberg correspondent à une orbite très grande de l'électron de valence, dont la taille peut valoir des milliers de fois celle de l'orbite fondamentale. Lorsque deux atomes, distants de quelques micromètres comme dans nos matrices, sont dans leur état fondamental, ils n'interagissent pas ${ }^{(d)}$. Ils interagissent au contraire fortement, même distants de quelques micromètres, lorsqu'ils sont tous les deux excités dans un état de Rydberg : cela provient de la grande séparation entre le noyau et l'électron de valence, qui conduit à un dipôle électrique très élevé.

Or, en physique quantique, on montre qu'un atome qui possède deux états peut être assimilé à un $\operatorname{spin} 1 / 2$ qui possède lui aussi deux états. On peut donc envisager de simuler des systèmes de spins en interaction sur réseau. Nous avons d'ailleurs fait quelques pas dans cette direction avec nos atomes de Rydberg [8]. Ces modèles de spins sont couramment utilisés en matière condensée pour modéliser, par exemple, les propriétés magnétiques de certains matériaux. De nombreuses questions restent ouvertes sur ces systèmes, du fait de l'impossibilité de calculer ab initio leurs propriétés lorsque le nombre de spins dépasse une trentaine dans des géométries de réseau triangulaire, par exemple [9] : c'est précisément ce que nous pouvons effectuer avec nos matrices d'atomes. Plus généralement, ces plans d'atomes permettent de réaliser l'ingénierie quantique de ces systèmes qui pourraient se révéler utiles pour le calcul quantique [10], ou la production d'états quantiques à la sensibilité exacerbée proposée pour la métrologie quantique. Mais tout ceci est une autre histoire...

\section{Remerciements}

Je souhaite adresser tous mes remerciements à mes collègues, Yvan Sortais, Thierry Lahaye et Philippe Grangier, ainsi qu'aux étudiants et postdoctorants qui se sont succédés sur ces expériences depuis une dizaine d'années, et dont la liste est malheureusement trop longue pour qu'ils soient cités ici. Ce sont pourtant eux qui font tout le travail expérimental...

(a) L'ouverture numérique d'une lentille de rayon $\mathrm{R}$ et de focale $\mathrm{f}$ est donnée approximativement par $\mathrm{ON} \approx \mathrm{R} / \mathrm{f}$ pour $\mathrm{R}<<\mathrm{f}$.

(b) En général, l'atome est perdu à la suite d'une collision sur l'un des atomes du gaz résiduel présent dans la chambre à vide, qui possède, lui, une vitesse très élevée correspondant à une température de $300 \mathrm{~K}$ environ.

(c) Voir encadré 2 de l'article de J.-M. Raimond dans ce numéro, p. 42

(d) La portée typique des interactions de van der Waals entre deux atomes dans leur état fondamental est de l'ordre de la taille de l'atome, soit environ $0,1 \mathrm{~nm}$. L'interaction est donc complètement négligeable à $1 \mu \mathrm{m}$ de distance.

\section{Références}

1• E. Schrödinger, British Journal of the Philosophy of Science 3, n'10 (1952) 109-123.

2• H. Dehmelt, Rev. Mod. Phys. 62 (1990) 525.

3• M. Leduc et P. Lemonde, Reflets de la physique n²1 (2010) 46-51.

4• S. Chu, C. Cohen-Tannoudji, W. D. Phillips, "Nobel Lectures", Rev. Mod. Phys. 70, n³ (1998) 685-741.

5• N. Schlosser et al., Nature (London) 411 (2001) 1024. 6• Y.R.P. Sortais et al., Phys. Rev. A 75 (2007) 013406.

7• F. Nogrette et al., Phys. Rev. X 4 (2014) 021034.

8• D. Barredo et al., Phys. Rev. Lett. 114 (2015) 113002.

9• M. Saffman et al., Rev. Mod. Phys. 82 (2010) 2313.

10• I.M. Georgescu et al., Rev. Mod. Phys. 86 (2014) 153. 